\title{
Pathogenicity of Fumonisin-producing and Nonproducing Strains of Aspergillus Species in Section Nigri to Maize Ears and Seedlings
}

\author{
G. P. Munkvold ${ }^{\dagger}$ and L. Weieneth, Iowa State University, Plant Pathology and Microbiology, Ames, IA; R. H. Proctor and M. Busman, \\ USDA-ARS, National Center for Agricultural Utilization Research, Peoria, IL; M. Blandino, Universita degli Studi di Torino, Italy; and \\ A. Susca, A. Logrieco, and A. Moretti, ISPA-CNR, Bari, Italy
}

\begin{abstract}
Species of Aspergillus section Nigri are commonly associated with maize kernels, and some strains can produce fumonisin mycotoxins. However, there is little information about the extent to which these fungi contribute to fumonisin contamination in grain, the damage they cause to maize ears, or their effects on maize seed germination and seedling health. We compared fumonisin-producing and nonproducing strains of A. niger, A. welwitschiae, A. phoenicis, A. tubingensis, and A. carbonarius from the United States and Italy in laboratory and field studies to assess their ability to contribute to fumonisin contamination, to cause maize ear rot, and to affect seed germination and seedling growth. In laboratory experiments, some strains of each Aspergillus species reduced germination or seedling growth, but there was high variability among strains within species. There were no consistent differences between fumonisin-producing and nonproducing strains. In field

studies in Iowa and Illinois, strains were variable in their ability to cause ear rot symptoms, but this was independent of the ability of the Aspergillus strains to produce fumonisins. Contamination of grain with fumonisins was not consistently increased by inoculation with Aspergillus strains compared with the control, and was much greater in $F$. verticillioides-inoculated treatments than in Aspergillus-inoculated treatments. However, the ratio of the $\mathrm{FB}$ analogs $\mathrm{FB}_{2}$ and $\mathrm{FB}_{1}$ was altered by inoculation with some Aspergillus strains, indicating that $\mathrm{FB}_{2}$ production by Aspergillus strains occurred in the field. These results demonstrate the pathogenic capabilities of strains of Aspergillus in section Nigri, but suggest that their effects on maize ears and seedlings are not related to their ability to produce fumonisins, and that fumonisin contamination of grain caused by Aspergillus spp. is not as significant as that caused by Fusarium spp.
\end{abstract}

The genus Aspergillus consists of over 200 species that are grouped into multispecies lineages known as sections (Prakash and Jha 2014). Section Nigri includes over 30 described species that are referred to as black aspergilli, or black Aspergillus species, and characterized by production of black asexual spores (conidia) (Hong et al. 2013; Varga et al. 2011). Black aspergilli can occur in diverse habitats, including in soil, on decaying organic matter, and within and on the surface of living plants. They can also cause food spoilage, and several species are associated with diseases in maize, peanut, grape, and onion (Palencia et al. 2010). Some black aspergilli can cause ear rot on maize and can also cause damage in storage if grain moisture content is sufficiently high (Koehler 1959). Black Aspergillus species are distantly related to aflatoxin-producing species (A. flavus and A. parasiticus), which also can cause maize ear rot and storage decay. In the United States, Aspergillus spp. are not known as severe seedling pathogens, although seedborne infection of up to $79 \%$ has been reported on maize (McGee 1988). A survey of untreated commercial seed lots in the U.S. reported an overall contamination of 2.8\% (Rodriguez-Brljevich 2008).

Some black Aspergillus species have a homolog of the fumonisin biosynthesis gene cluster that was first characterized in the maize pathogen Fusarium verticillioides. Fumonisins are of concern to agriculture and food/feed safety because they are epidemiologically associated with some human diseases and they cause multiple diseases in domestic animals. Species of Aspergillus do not possess orthologs of all genes in the Fusarium fumonisin cluster; in particular, they lack an ortholog of the Fusarium gene FUM2, which is required for production of the fumonisin analogs fumonisins $\mathrm{B}_{1}\left(\mathrm{FB}_{1}\right)$ and $\mathrm{B}_{3}\left(\mathrm{FB}_{3}\right)$, but not the analog fumonisin $\mathrm{B}_{2}\left(\mathrm{FB}_{2}\right)$ (Susca et al. 2010). Among black Aspergillus species, fumonisin production was first reported in Aspergillus niger in 2007 (Frisvad et al. 2007). In this and in most

\section{${ }^{\dagger}$ Corresponding author: G. P. Munkvold; E-mail: munkvold@iastate.edu}

*The $\boldsymbol{e}$-Xtra logo stands for "electronic extra" and indicates that one supplementary figure is published online.

Accepted for publication 7 September 2017.

(C) 2018 The American Phytopathological Society subsequent reports, $A$. niger strains were observed to produce $\mathrm{FB}_{2}$, but not $\mathrm{FB}_{1}$ or $\mathrm{FB}_{3}$, observations that are consistent with the absence of a FUM2 ortholog in the Aspergillus fumonisin cluster. This production profile contrasts with the high concentrations of $\mathrm{FB}_{1}$ and lower concentrations of $\mathrm{FB}_{2}$ and $\mathrm{FB}_{3}$ production typically reported for Fusarium spp. Some strains of Aspergillus isolated from raisins have been reported to produce $\mathrm{FB}_{1}$ and $\mathrm{FB}_{3}$ (Varga et al. 2010), but this finding has been disputed (Nielsen and Logrieco 2012a, b; Varga et al. 2012). Production of two additional fumonisin analogs has also been reported in $A$. niger: $\mathrm{FB}_{4}$, which is also produced at low concentrations by Fusarium (Noonim et al. 2009), and $\mathrm{FB}_{6}$, which has the same elemental composition as $\mathrm{FB}_{1}$, but differs in structure (Månsson et al. 2010).

It is unclear how fumonisin production benefits fumonisinproducing fungi. In Fusarium, the role of fumonisins in pathogenicity is controversial. Fumonisins are not necessary for pathogenicity, as is evident by the ability of nonproducing strains to infect maize and cause ear rot (Desjardins et al. 2002). However, in a study by Desjardins et al. (1995), nearly all highly virulent strains also produced high concentrations of $\mathrm{FB}_{1}$, while many less virulent strains did not. Additionally, disease symptoms and stunting can be induced in maize seedlings by fumonisins alone, although the seedlings eventually outgrew the effects at the lower concentrations tested (Arias et al. 2012). Maize seedlings have some ability to detoxify low concentrations of fumonisin, but greater concentrations have detrimental effects, possibly by inducing premature senescence through their interference with sphingolipid metabolism (Arias et al. 2012). In A. niger, blocking $\mathrm{FB}_{2}$ production by disruption of the fum 8 gene, which encodes an enzyme that catalyzes an early step in fumonisin biosynthesis, does not affect vegetative growth, sensitivity to temperature, or sensitivity to UV light, suggesting it does not play a role in normal growth of the fungus (Shimizu et al. 2015).

It is possible that fumonisins contribute to virulence or pathogenicity in Aspergillus species, but this has not yet been experimentally tested. The current study was intended to examine this question by comparing the virulence of a range of fumonisin-producing and nonproducing strains of Aspergillus section Nigri. We designed our study to compare a wide selection of strains from Aspergillus section Nigri, including both fumonisin-producing and nonproducing strains. Warm and cold germination assays were used to evaluate seed germination under ideal and cold stress conditions, and rolled-towel assays were 
used to measure effects of inoculation on seedling growth. Field studies were conducted in Iowa and Illinois to assess ear rot development and the effects of inoculation on fumonisin contamination of grain.

\section{Materials and Methods}

Germination and seedling disease assays. Three types of assays were conducted to assess the effects of Aspergillus strains on maize seed and seedlings: a warm germination assay, a cold germination assay (AOSA rules), and a rolled-towel assay (Ellis et al. 2011). Twenty-six strains of black Aspergillus were obtained from maize kernel samples from Iowa, Idaho, Illinois, and Italy, and were selected to provide a broad representation of black Aspergillus species occurring on maize, including fumonisin-producing and nonproducing strains, as determined in a previous study (Susca et al. 2014) (Tables 1,2, and 3). The strains had initially been placed in Aspergillus section Nigri based on morphological characteristics, and were further identified to species based on $\beta$-tubulin and calmodulin gene sequences (Susca et al. 2014).

Each Aspergillus strain was grown on Difco potato dextrose agar (PDA) (Becton, Dickinson, and Co., Sparks, MD) under fluorescent light at 20 to $25^{\circ} \mathrm{C}$ for 7 days. The spores were rinsed off the plates, gently dislodging them from the colonies, and suspended in sterile distilled water (SDW). The spore concentrations of the suspensions were determined using a hemocytometer, and were diluted to a final concentration of $10^{6}$ spores $/ \mathrm{ml}$. For all spore suspensions and control treatments, Tween 80 was added as a surfactant at a concentration of 0.125 to $0.5 \mathrm{ml}$ per liter of SDW. Fresh suspensions were prepared for each run of each experiment, all with the same spore concentration, except in the second runs of the germination assays, one strain (ITEM 15167) produced insufficient spores to reach the necessary volume of $10^{6}$ spores $/ \mathrm{ml}$, so $5 \times 10^{5}$ was used instead. In the first run of the warm germination experiment, two strains, ITEM 15065 and NRRL 62526, were not included because of insufficient spore production; in the second run, ITEM 15099 was not included, for the same reason.

Two maize hybrids were used in the two repetitions of the warm germination, cold, and rolled-towel assays. Hybrid A was an experimental hybrid obtained from Syngenta, and hybrid B was Syngenta hybrid 85v88-3000GT (Syngenta Seeds, Northfield, MN). The maize kernels were first surface disinfested by submerging for $5 \mathrm{~min}$ in $0.6 \%$ sodium hypochlorite, followed by $3 \mathrm{~min}$ in $75 \%$ ethanol, and finally $2 \mathrm{~min}$ in sterile water (all steps at room temperature). The kernels were then added to flasks containing the spore suspensions (or the control of Tween water), and placed on a shaker at room temperature $\left(20\right.$ to $25^{\circ} \mathrm{C}$ ) for $12 \mathrm{~h}$ at $130 \mathrm{rpm}$ for the first run, and $80 \mathrm{rpm}$ for the second. For the first run of the warm and cold germination tests, 820 seeds were inoculated for each treatment, divided between two $250-\mathrm{ml}$ flasks with $100 \mathrm{ml}$ of suspension in each. For the second run, 850 seeds were placed in a single 500-ml flask, with $300 \mathrm{ml}$ suspension. After $12 \mathrm{~h}$, the suspension was drained off, and the kernels spread on paper towels to dry in a biosafety hood.

Table 1. Effects of seed inoculation with fumonisin-producing or nonproducing Aspergillus strains on seedling growth in a laboratory rolled-towel assay, ${ }^{\mathrm{x}} \mathrm{U} . \mathrm{S}$. strains, hybrid A

\begin{tabular}{|c|c|c|c|c|c|c|c|}
\hline Strain & Origin & Species & $\mathbf{F B}_{2}{ }^{\mathbf{y}}$ & Root weight $(\mathrm{g})^{\mathrm{z}}$ & Shoot weight $(\mathrm{g})^{\mathrm{z}}$ & Root length $(\mathrm{cm})^{\mathrm{z}}$ & Shoot length $(\mathrm{cm})^{\mathrm{z}}$ \\
\hline Control & NA & NA & NA & 1.85 & 5.24 & 13.22 & 9.69 \\
\hline ENDO 3233 & Illinois & A. niger & + & 1.33 & $3.06^{*}$ & 16.42 & $5.80 *$ \\
\hline NRRL 62522 & Illinois & A. phoenicis & + & $1.12^{*}$ & $2.52 *$ & 13.33 & $5.00 *$ \\
\hline NRRL 62526 & Illinois & A. phoenicis & + & $1.00^{*}$ & $2.28 *$ & 12.20 & $4.44 *$ \\
\hline ITEM 15304 & Iowa & A. tubingensis & - & $0.87 *$ & $1.89^{*}$ & 9.95 & $3.38 *$ \\
\hline ITEM 15309 & Idaho & A. welwitschiae & + & 2.05 & 5.00 & 14.47 & 9.26 \\
\hline ITEM 15318 & Iowa & A. tubingensis & - & 1.43 & 3.93 & 15.73 & $6.85^{*}$ \\
\hline ITEM 15335 & Iowa & A. welwitschiae & + & 1.36 & $2.99 *$ & 15.91 & $5.21 *$ \\
\hline ITEM 15337 & Iowa & A. niger & + & 1.48 & $2.92 *$ & 13.21 & $5.43 *$ \\
\hline ITEM 15349 & Iowa & A. tubingensis & - & 1.42 & $3.56^{*}$ & 15.76 & $5.87 *$ \\
\hline ITEM 15375 & Iowa & A. niger & - & 1.72 & $3.51 *$ & 15.47 & $6.40 *$ \\
\hline \multicolumn{4}{|c|}{$P$-value for $\mathrm{FB}_{2}+$ versus $\mathrm{FB}_{2}-$ comparison } & 0.7985 & 0.7303 & 0.8359 & 0.7555 \\
\hline
\end{tabular}

${ }^{x}$ Seeds were inoculated with $0.1 \mathrm{ml}$ of a spore suspension $\left(10^{6}\right.$ spores $\left./ \mathrm{ml}\right)$ and incubated in rolled, damp paper towels under ambient conditions $\left(20\right.$ to $\left.25^{\circ} \mathrm{C}\right)$ for 1 week.

${ }^{y}$ The + or - designation refers to whether or not the isolate produces fumonisin $\mathrm{FB}_{2}$.

${ }^{\mathrm{z}}$ Values are means of three replications of 15 seedlings each. The asterisk $\left(^{*}\right)$ indicates treatments significantly different from the noninoculated control, according to Fisher's protected least significant difference $(\alpha=0.05)$.

Table 2. Effects of seed inoculation with fumonisin-producing or nonproducing Aspergillus strains on seedling growth following inoculation with a spore suspension of each of the Aspergillus species strains collected from Italy in a laboratory rolled-towel assay ${ }^{\mathrm{x}}$ on an experimental corn hybrid (hybrid A)

\begin{tabular}{|c|c|c|c|c|c|c|c|}
\hline Strain & Origin & Species & $\mathrm{FB}_{2}{ }^{\mathrm{y}}$ & Root weight $(\mathrm{g})^{\mathrm{z}}$ & Shoot weight $(\mathrm{g})^{\mathrm{z}}$ & Root length $(\mathrm{cm})^{\mathrm{z}}$ & Shoot length $(\mathrm{cm})^{\mathrm{z}}$ \\
\hline Control & NA & NA & NA & 2.61 & 6.33 & 15.88 & 10.94 \\
\hline ITEM 15065 & Marche & A. carbonarius & - & $1.75^{*}$ & $3.54 *$ & 16.84 & $5.65^{*}$ \\
\hline ITEM 15078 & Marche & A. niger & + & $2.15^{*}$ & $3.79 *$ & 15.92 & $6.50 *$ \\
\hline ITEM 15096 & Veneto & A. niger & - & $1.73 *$ & $3.38 *$ & 18.05 & $6.06^{*}$ \\
\hline ITEM 15099 & Veneto & A. niger & + & $1.64 *$ & $3.60 *$ & 17.55 & $6.12 *$ \\
\hline ITEM 15114 & Veneto & A. niger & - & $1.64 *$ & $2.72 *$ & $18.27 *$ & $4.83 *$ \\
\hline ITEM 15129 & Veneto & A. welwitschiae & - & $1.79 *$ & $4.25 *$ & $19.38^{*}$ & $6.48^{*}$ \\
\hline ITEM 15132 & Veneto & A. welwitschiae & + & $1.77 *$ & $3.91 *$ & $18.74^{*}$ & $6.53 *$ \\
\hline ITEM 15165 & Piemonte & A. niger & + & $3.24 *$ & 6.29 & $20.28^{*}$ & 10.70 \\
\hline ITEM 15167 & Lombardia & A. niger & + & $1.66^{*}$ & $4.08^{*}$ & 18.24 & $7.11^{*}$ \\
\hline ITEM 15206 & E.-Romagna & A. niger & + & $1.43^{*}$ & $3.28 *$ & 15.98 & $5.21 *$ \\
\hline ITEM 15225 & Molise & A. niger & - & $2.16^{*}$ & $3.46^{*}$ & 17.87 & $5.82 *$ \\
\hline \multicolumn{4}{|c|}{$P$-value for $\mathrm{FB}_{2}+$ versus $\mathrm{FB}_{2}-$ comparison } & 0.3665 & 0.0412 & 0.6318 & 0.0324 \\
\hline
\end{tabular}

${ }^{x}$ Seeds were inoculated with $0.1 \mathrm{ml}$ of a spore suspension $\left(10^{6}\right.$ spores $\left./ \mathrm{ml}\right)$ and incubated in rolled, damp paper towels under ambient conditions $\left(20\right.$ to $\left.25^{\circ} \mathrm{C}\right)$ for 1 week.

${ }^{y}$ The + or - designation refers to whether or not the isolate produces fumonisin $\mathrm{FB}_{2}$.

${ }^{\mathrm{z}}$ Values are means of three replications of 15 seedlings each. The asterisk $(*)$ indicates treatments significantly different from the noninoculated control, according to Fisher's protected least significant difference $(\alpha=0.05)$. 
After the kernels had dried, they were submitted to the Iowa State University Seed Testing Laboratory for a standard warm germination test (AOSA 2014), which includes qualitative visual observation of fungal sporulation, and a cold test (AOSA 2014). In the warm germination test, the seeds were grown on moist blotter paper at $25^{\circ} \mathrm{C}$, and evaluated after 1 week for the percentage germination. Seedlings were scored as normal, abnormal, or dead, and the percentage of normal seedlings was considered to be percent germination. The cold test approximates stresses of early spring planting by covering seeds with a layer of moist, nonsterilized sand-soil mixture, chilling for 1 week at $10^{\circ} \mathrm{C}$, and then warming up to $25^{\circ} \mathrm{C}$ for an additional week before evaluation of germination. Percent germination was considered to be the percent of seedlings that emerged from the sand-soil mixture. For both assays, four 100-seed replicates were used for each combination of Aspergillus strain and hybrid, including noninoculated control treatments.

Maize seeds of hybrids A and B were also used for the rolled-towel assay. Each replicate consisted of 15 seeds, with three replicates for each treatment. The assay was conducted once with each hybrid. In the first run (hybrid A), the U.S. strains were run as one experiment, and the Italian strains were run as a second, due to logistical considerations. In the second run (hybrid B), all strains were run in the same experiment. To inoculate, the 15 surface-disinfested seeds were placed two thirds of the way up on two layers of damp paper towel, and $0.1 \mathrm{ml}$ of a spore suspension (or the control of Tween water) was then pipetted over the top of each seed. Seeds were then covered with a third damp towel, and the layered towels were loosely rolled and placed in individual unsealed plastic bags. These bags were set upright in 5-gallon (18.9-liter) buckets so that the rolled towels were vertical, and a clear plastic bag was placed over the top of each bucket to prevent contamination or drying. After 7 days of incubation under ambient conditions (fluorescent lighting, 20 to $25^{\circ} \mathrm{C}$ ), seed germination was evaluated by examining growth of the resulting seedlings. The lengths of the longest root and shoot were measured on each seedling, at the point of the furthest distance from the seed. The roots and shoots were separated and weighed immediately after unwrapping each replicate, to avoid drying. The seed itself was not weighed, so nongerminated seeds were recorded as 0 for both weights. For all measurements, the values for the 15 seedlings on each towel were averaged and analyzed as a single observation. Samples of root tissue were taken from a few arbitrarily selected seedlings in each treatment to recover the inoculated Aspergillus strains on PDA.

All data were subject to analysis of variance using PROC GLM (SAS version 9.1). Fisher's protected least significant difference (LSD) was used to compare treatments, with the level of significance $P<0.05$. Data for some strains were missing in each experiment due to inadequate spore production.

Field experiments. Experiments were conducted in Story Co., Iowa, in 2012 and 2013, and in Peoria Co., Illinois, in 2012. The experimental design was similar at both locations, but specific inoculation methods and fungal strains differed slightly.

Iowa field experiments were conducted in a single location in both years with a single maize hybrid. Trials were planted in mid-April 2012 and mid-June 2013 in a reduced-tillage field where maize was the previous crop. Standard commercial fungicide/insecticide seed treatments were used, but no other fungicides or insecticides were applied. Weed control was accomplished by two applications of glyphosate during the early growth stages. Field plots consisted of single rows spaced $75 \mathrm{~cm}$ apart by $5.2 \mathrm{~m}$ in length, with approximately 35 seeds per row. At silk emergence, five ears per row were arbitrarily selected and tagged for inoculation. Two types of inoculation were carried out, as described by Reid et al. (1996): silk inoculation and wound inoculation. For the silk inoculation, $2 \mathrm{ml}$ of a spore

Table 3. Effects of seed inoculation with fumonisin-producing or nonproducing Aspergillus strains on seedling growth following inoculation with a spore suspension of each of the Aspergillus species strains collected from the U.S. or Italy in a laboratory rolled-towel assay on an experimental corn hybrid (hybrid B, Syngenta 85v88-3000GT)

\begin{tabular}{|c|c|c|c|c|c|c|c|}
\hline Strain & Origin & Species & $\mathrm{FB}_{2}{ }^{\mathrm{y}}$ & Root weight $(g)^{z}$ & Shoot weight $(g)^{z}$ & Root length $(\mathrm{cm})^{\mathrm{z}}$ & Shoot length $(\mathrm{cm})^{\mathrm{z}}$ \\
\hline Control & NA & NA & NA & 4.45 & 4.89 & 18.45 & 8.26 \\
\hline ENDO 3233 & Illinois & A. niger & + & $1.81 *$ & $2.10^{*}$ & $12.33 *$ & $3.92 *$ \\
\hline NRRL 62518 & Illinois & A. niger & + & $1.55^{*}$ & $1.98 *$ & $12.13^{*}$ & $4.09 *$ \\
\hline NRRL 62522 & Illinois & A. phoenicis & + & $2.10 *$ & $2.48 *$ & 15.32 & $5.00 *$ \\
\hline NRRL 62526 & Illinois & A. phoenicis & + & $2.04 *$ & $2.02 *$ & 15.12 & $4.21 *$ \\
\hline ITEM 15304 & Iowa & A. tubingensis & - & $2.81 *$ & $3.13 *$ & 16.29 & $5.46^{*}$ \\
\hline ITEM 15309 & Idaho & A. welwitschiae & + & $3.46 *$ & $3.80 *$ & 16.43 & 7.44 \\
\hline ITEM 15318 & Iowa & A. tubingensis & - & $2.78 *$ & $3.54 *$ & 18.02 & $6.39 *$ \\
\hline ITEM 15330 & Iowa & A. tubingensis & - & $2.04 *$ & $2.50 *$ & $12.54 *$ & $4.61 *$ \\
\hline ITEM 15335 & Iowa & A. welwitschiae & + & $2.35 *$ & $2.51 *$ & 16.00 & $4.86^{*}$ \\
\hline ITEM 15337 & Iowa & A. niger & + & $1.79 *$ & $1.99 *$ & $13.88 *$ & $4.16^{*}$ \\
\hline ITEM 15349 & Iowa & A. tubingensis & - & $2.51 *$ & $3.36^{*}$ & 15.61 & $5.81 *$ \\
\hline ITEM 15353 & Iowa & A. niger & + & $2.36^{*}$ & $2.22 *$ & $14.88 *$ & $4.87 *$ \\
\hline ITEM 15375 & Iowa & A. niger & - & $2.51 *$ & $2.27 *$ & $14.26^{*}$ & $4.54 *$ \\
\hline ITEM 15065 & Marche & A. carbonarius & - & 4.06 & 4.25 & 18.77 & 8.04 \\
\hline ITEM 15078 & Marche & A. niger & + & $2.22 *$ & $1.69 *$ & $14.18 *$ & $3.26 *$ \\
\hline ITEM 15096 & Veneto & A. niger & - & $1.91 *$ & $1.82 *$ & $13.37 *$ & $3.86^{*}$ \\
\hline ITEM 15114 & Veneto & A. niger & - & $1.89^{*}$ & $1.78 *$ & $13.29 *$ & $3.73 *$ \\
\hline ITEM 15129 & Veneto & A. welwitschiae & - & $1.93 *$ & $1.96 *$ & $11.11 *$ & $3.87 *$ \\
\hline ITEM 15132 & Veneto & A. welwitschiae & + & $2.13^{*}$ & $2.49 *$ & $14.34 *$ & $5.04 *$ \\
\hline ITEM 15165 & Piemonte & A. niger & + & $2.08 *$ & $2.41 *$ & $13.67 *$ & $4.68 *$ \\
\hline ITEM 15167 & Lombardia & A. niger & + & $2.82 *$ & 3.85 & 18.01 & 7.30 \\
\hline ITEM 15178 & E.-Romagna & A. niger & - & $2.18 *$ & $2.39 *$ & 15.58 & $4.80 *$ \\
\hline ITEM 15187 & E.-Romagna & A. welwitschiae & - & $3.16^{*}$ & $3.62 *$ & 19.34 & $6.50 *$ \\
\hline ITEM 15206 & E.-Romagna & A. niger & + & $2.21 *$ & $2.32 *$ & $14.65 *$ & $4.37 *$ \\
\hline ITEM 15225 & Molise & A. niger & - & $3.53 *$ & 3.87 & 19.26 & 6.90 \\
\hline \multicolumn{4}{|c|}{$P$-value for $\mathrm{FB}_{2}+$ versus $\mathrm{FB}_{2}-$ comparison } & 0.0313 & 0.0363 & 0.1565 & 0.1498 \\
\hline
\end{tabular}

${ }^{x}$ Seeds were inoculated with $0.1 \mathrm{ml}$ of a spore suspension $\left(10^{6} \mathrm{spores} / \mathrm{ml}\right)$ and incubated in rolled, damp paper towels under ambient conditions $\left(20\right.$ to $\left.25^{\circ} \mathrm{C}\right)$ for 1 week.

y The + or - designation refers to whether or not the isolate produces fumonisin $\mathrm{FB}_{2}$.

${ }^{z}$ Values are means of three replications of 15 seedlings each. The asterisk $(*)$ indicates treatments significantly different from the noninoculated control, according to Fisher's protected least significant difference $(\alpha=0.05)$. 
suspension $\left(10^{6}\right.$ spores $/ \mathrm{ml}$, as previously described) was injected by hypodermic needle into the silk channel of the selected ears, 7 to 10 days after silk emergence, without wounding the kernels or cob. For the wound inoculation, ears were wounded by pressing a pinbar against the side of the ear, approximately 21 days after silk emergence, followed immediately by injection through the husk of $2 \mathrm{ml}$ of a spore suspension into the center of the wounded area. The two inoculation types were applied in separate, adjacent experiments in both years. Within each inoculation type experiment, there were seven treatments, arranged in a randomized complete block design. Treatments consisted of inoculation with one of five strains of black Aspergillus, inoculation with a strain of $F$. verticillioides, or a mockinoculation with SDW containing Tween. The Aspergillus strains used in the studies were ITEM 15309, 15335, 15337, 15353, and 15375 (Table 1); the $F$. verticillioides strain was ITEM 3927, a fumonisin-producing strain from Iowa maize. Following inoculation, the ears were left uncovered; after the plants matured and dried in the field to approximately $20 \%$ kernel moisture content, ears were collected, brought to the laboratory, and scored for ear rot severity using a 1 to 7 scale (Reid et al. 1996), in which $1=$ no symptoms, $2=$ up to $3 \%$ ear rot severity, $3=4$ to $10 \%, 4=11$ to $25 \%, 5=26$ to $50 \%, 6=51$ to $75 \%$, and $7=76$ to $100 \%$. A few arbitrarily selected symptomatic kernels were collected from each treatment to recover the inoculated Aspergillus strains on PDA. After scoring, ears were dried in a forced air grain dryer at $38^{\circ} \mathrm{C}$ until grain moisture was $<13 \%$. Ears were then hand-shelled and the kernels were ground using a Romer mill (Model 2A, Romer Labs, Washington, MO, U.S.A.).

The Illinois field experiment was conducted in a single location in Peoria Co., IL, in 2012 with a single maize inbred line, B73. Trials were planted on 16 May 2012 in plots where wheat was the previous crop. Neither commercial fungicide/insecticide seed treatments, nor other fungicides or insecticides were used. Weed control was accomplished by a single spot application of 2,4-D/dicamba during the early growth stage of the crop and by cultivation. Field plots were composed of rows spaced $100 \mathrm{~cm}$ apart by $20 \mathrm{~m}$ in length, with approximately 120 seeds per row. At silk emergence, 30 ears per row were arbitrarily selected and tagged for inoculation. Two types of inoculation were carried out: silk inoculation as described by Reid et al. (1996), and wound inoculation as described by Dowell et al. (2002). For the wound inoculation, ears were wounded by making a 4-cm incision into the side of the ear, approximately 21 days after silk emergence, followed immediately by insertion through the husk of an $8-\mathrm{cm}$ sterile pipe cleaner, saturated in a spore suspension $\left(10^{6}\right.$ spores $/ \mathrm{ml}$, as previously described), into the wounded area. The two inoculation types were applied in separate, adjacent experiments. Within each inoculation type experiment, there were six treatments. Treatments consisted of inoculation with one of five strains of black Aspergillus species, or a mock-inoculation with sterile distilled water. Aspergillus strains used in the studies were ITEM 15309, ITEM 15337, ITEM 15353, and ITEM 15375 (Table 1), and ITEM 15333, a fumonisin-producing strain of $A$. niger from maize grown in Iowa. Following inoculation, methods were followed as described for the Iowa experiments.

Grain from the Iowa and Illinois field experiments was analyzed for $\mathrm{FB}_{1}, \mathrm{FB}_{2}$, and $\mathrm{FB}_{3}$ by LC-MS/MS at the NCAUR laboratory in Peoria, IL. All grain from each field plot was ground, and 10-g subsamples were extracted with $50 \mathrm{ml}$ of acetonitrile/water $(1: 1, \mathrm{v} / \mathrm{v})$. Maize solvent slurries were allowed to steep for $2 \mathrm{~h}$, with gentle shaking, and then extracts were filtered through a Whatman $2 \mathrm{~V}$ filter. The analytical method utilized a LC-MS/MS instrument consisting of a ThermoFisher (Sunnyvale, CA, U.S.A.) UltiMate 3000 ultra-high performance liquid chromatography (UPLC) system and an ABSCIEX (Framingham, MA, U.S.A.) QTRAP 3200 mass spectrometer. The mass spectrometer was operated in positive mode utilizing an electrospray ionization (ESI) interface. Injections of $10 \mu \mathrm{l}$ of analyte were eluted from a Phenomenex (Torrance, CA, U.S.A.) Kinetex XB-C18 $2.1 \times 50 \mathrm{~mm}$ column with a $600 \mu \mathrm{l} / \mathrm{min}$ gradient flow of water/methanol $(\mathrm{MeOH})$. Approximately $10 \%$ of the column flow was directed to the ESI interface of the mass spectrometer. UPLC solvents were acidified with $0.3 \%$ acetic acid. The gradient program consisted of the following steps: 0 to $1 \mathrm{~min}, 40 \% \mathrm{MeOH}$; 1 to $11 \mathrm{~min}$, 40 to $95 \% \mathrm{MeOH} ; 11$ to $13 \min , 95 \% \mathrm{MeOH} ; 13$ to $14 \mathrm{~min}, 95$ to $40 \% \mathrm{MeOH}$; and 14 to $15 \mathrm{~min}, 40 \% \mathrm{MeOH}$. ESI-MS/MS detection of fumonisins was accomplished by monitoring characteristic fragment ions $\left(\mathrm{FB}_{1}: \mathrm{m} / \mathrm{z} 352,528\right.$; and $\left.\mathrm{FB}_{2}, \mathrm{FB}_{3}: \mathrm{m} / \mathrm{z} 336,512\right)$ from the $\mathrm{m} / \mathrm{z} 722\left(\mathrm{FB}_{1}\right)$ and $706\left(\mathrm{FB}_{2}, \mathrm{FB}_{3}\right)[\mathrm{M}+\mathrm{H}]^{+}$ions of the fumonisins in multiple reaction monitoring mode. Quantitation of fumonisins was done on the basis of the integrated intensity of the $\mathrm{m} / \mathrm{z} 352$ and 336 fragments compared with a calibration curve generated from fumonisin standard solutions. $\mathrm{FB}_{2}$ and $\mathrm{FB}_{3}$ were distinguished by chromatographic retention time.

Data were analyzed using ANOVA (SAS ver. 9.4 or SAS Enterprise ver. 7.1, PROC GLM). Fisher's protected least significant difference (LSD) was used to compare treatments, with the level of significance $P<0.05$. Prior to data analysis, ear rot severity scores were transformed to percentages by converting the 1 to 7 scores to the midpoint of the percentage range of each score. The mean percentage of symptomatic kernels was calculated for the five ears from each plot, and those mean values were subjected to ANOVA. Data were analyzed separately for silk-channel and wound inoculations, because these inoculation treatments were done in separate experiments. Grain from the four replicate plots receiving the silk-channel inoculation in the Illinois field experiment were combined prior to fumonisin analysis; therefore, there are no statistical replications for this treatment and these data were omitted from the ANOVA.

\section{Results}

Germination assays. Aspergillus strains varied substantially in their effects on warm germination and cold test results for both hybrids. Several strains differed significantly from the noninoculated control in each test. Some strains ranked more consistently near the high or low end in all four tests, while the ranking of other strains varied widely among experiments. The presence of black Aspergillus sporulation was observed in all inoculated treatments, but not in the water control treatments. For hybrid A, the warm germination percentage varied from approximately $75 \%$ to nearly $100 \%$, with the control near the high end of the range at $96.5 \%$ (Fig. 1). Twelve of the 24 strains reduced warm germination significantly compared with the control. Among the 13 fumonisin-producing strains, seven reduced warm germination significantly, with means ranging from $88.3 \%$ down to $81.9 \%$, and the others had no significant effect. In the cold germination test, the differences were even more pronounced, with cold test germination ranging from less than $40 \%$ to over $80 \%$ (Fig. 2). In this test, the control was near the middle of the range at $62.5 \%$, with some strains of Aspergillus resulting in significantly greater cold test germination than the control. Eleven of the 26 strains significantly reduced cold test germination compared with the control (means ranging from 39.0 to $51.6 \%$ ), 11 strains did not differ from the control, and four of the 26 strains had cold germination values significantly greater than the control in the range of 75.0 to $82.8 \%$. Among the 14 fumonisin-producing strains, seven reduced cold test germination, five did not differ from the control, and two were significantly greater than the control.

In hybrid $\mathrm{B}$, in the warm germination test results were much lower overall, but still displayed a wide variation among treatments, from a minimum of approximately $45 \%$, to the control at $71.0 \%$ (Fig. $3)$. Nineteen of the 25 Aspergillus strains significantly reduced warm germination compared with the control. Eleven of the 13 fumonisin-producing strains significantly reduced germination compared with the control, with a mean of $45.3 \%$ for the low (ITEM 15078). Hybrid B generally performed poorly in the cold test, with no treatment resulting in cold test germination greater than $60 \%$, and some near $40 \%$ (Fig. 4). The control was at the lower end of the range at $49.8 \%$, but only four of the 25 strains had values significantly greater than the control (up to $57.7 \%$ ). For cold germination, only two of the 25 strains (both fumonisin-producing) had values significantly lower than the control, with means of 41.6 and $42.3 \%$. Among the 13 fumonisin-producing strains, two reduced cold test germination, 10 did not differ from the control, and one was significantly greater than the control. 


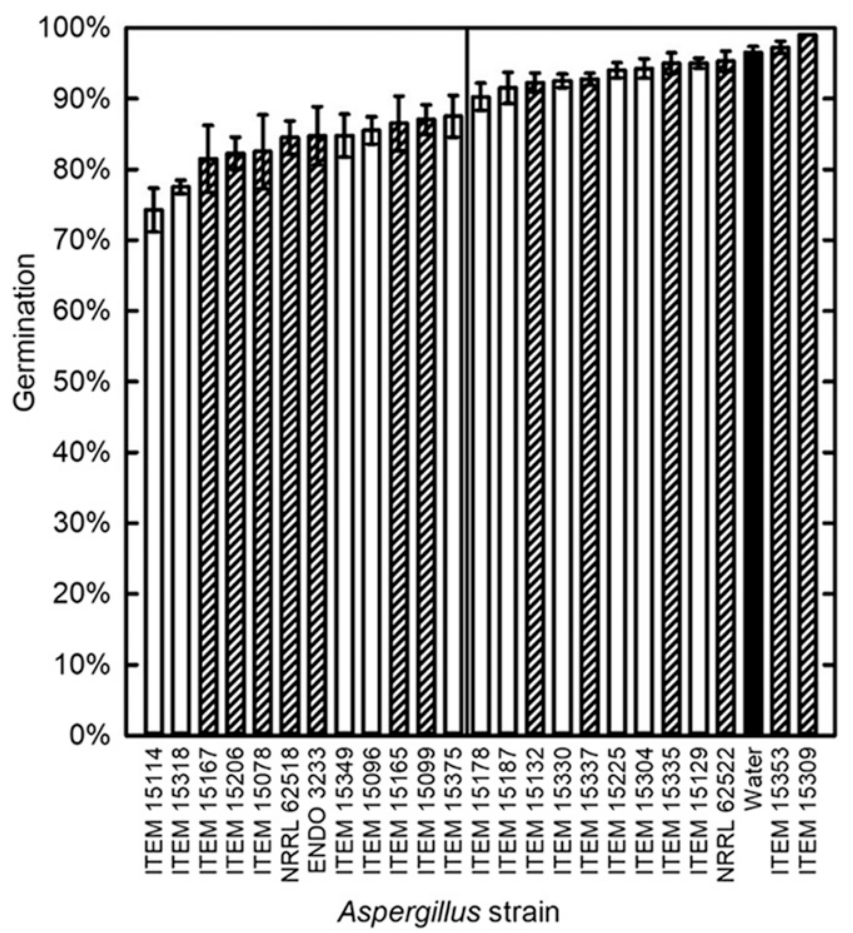

Fig. 1. Germination percentages in warm germination test for maize kernels (hybrid $A$ ) inoculated with strains of Aspergillus with and without production of fumonisin $\mathrm{B}_{2}$. Hatch marks indicate strains that have previously been documented to produce fumonisin $B_{2}$. Strains to the right of the vertical line were not significantly different from the water control $(\mathrm{LSD}=6.9 \%, \alpha=0.05)$. Error bars represent the standard error of the mean.

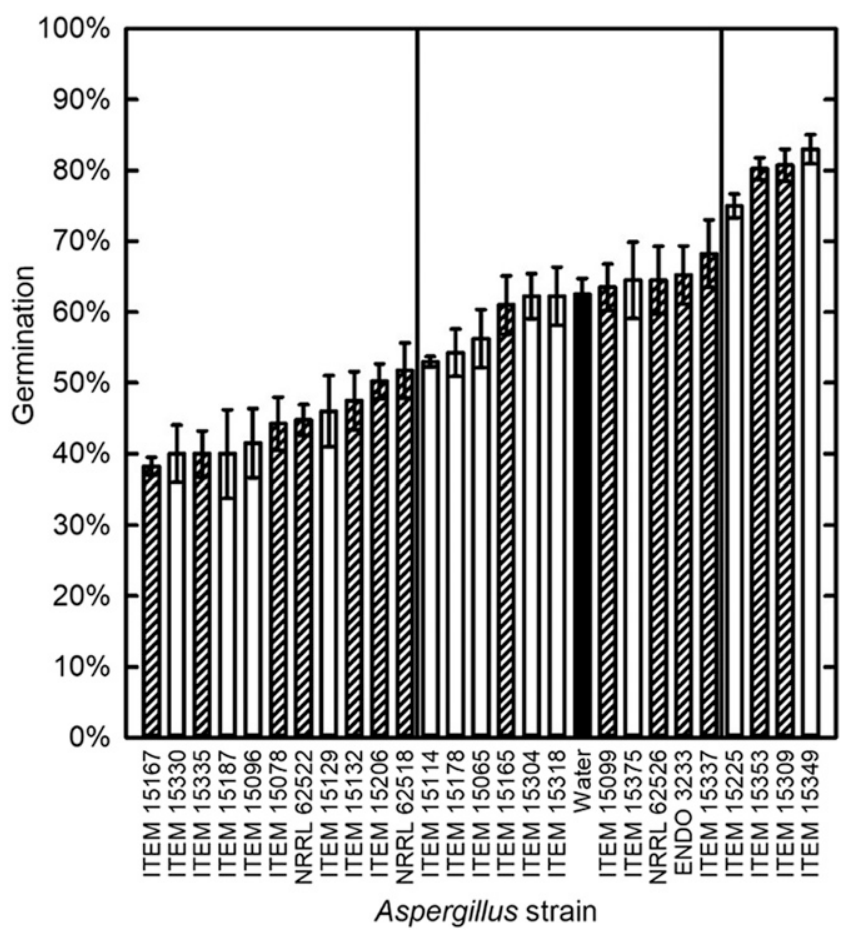

Fig. 2. Germination percentages in cold germination test for maize kernels (hybrid A) inoculated with strains of Aspergillus with and without production of fumonisin $\mathrm{B}_{2}$. Hatch marks indicate strains that have previously been documented to produce fumonisin $B_{2}$. Strains to the right of the vertical line were not significantly different from the water control (LSD $=10.4 \%, \alpha=0.05)$. Error bars represent the standard error of the mean.

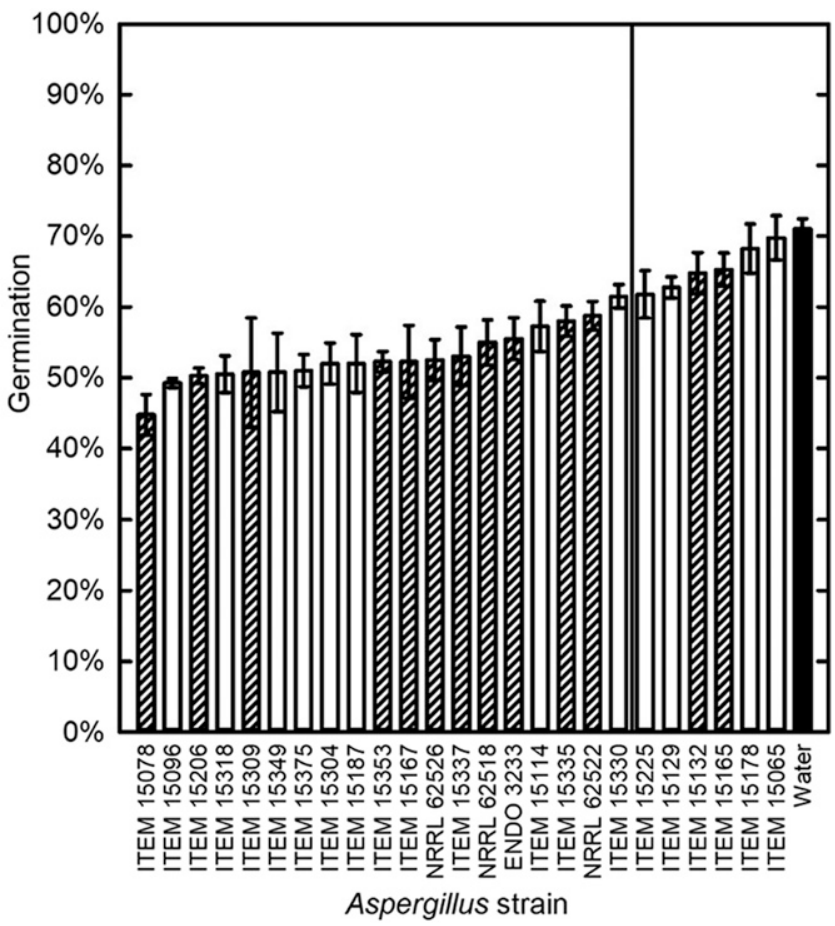

Fig. 3. Germination percentages in warm germination test for maize kernels (hybrid B) inoculated with strains of Aspergillus with and without production of fumonisin $B_{2}$. Hatch marks indicate strains that have previously been documented to produce fumonisin $B_{2}$. Strains to the right of the vertical line were not significantly different from the water control $(\mathrm{LSD}=9.3 \%, \alpha=0.05)$. Error bars represent the standard error of the mean.

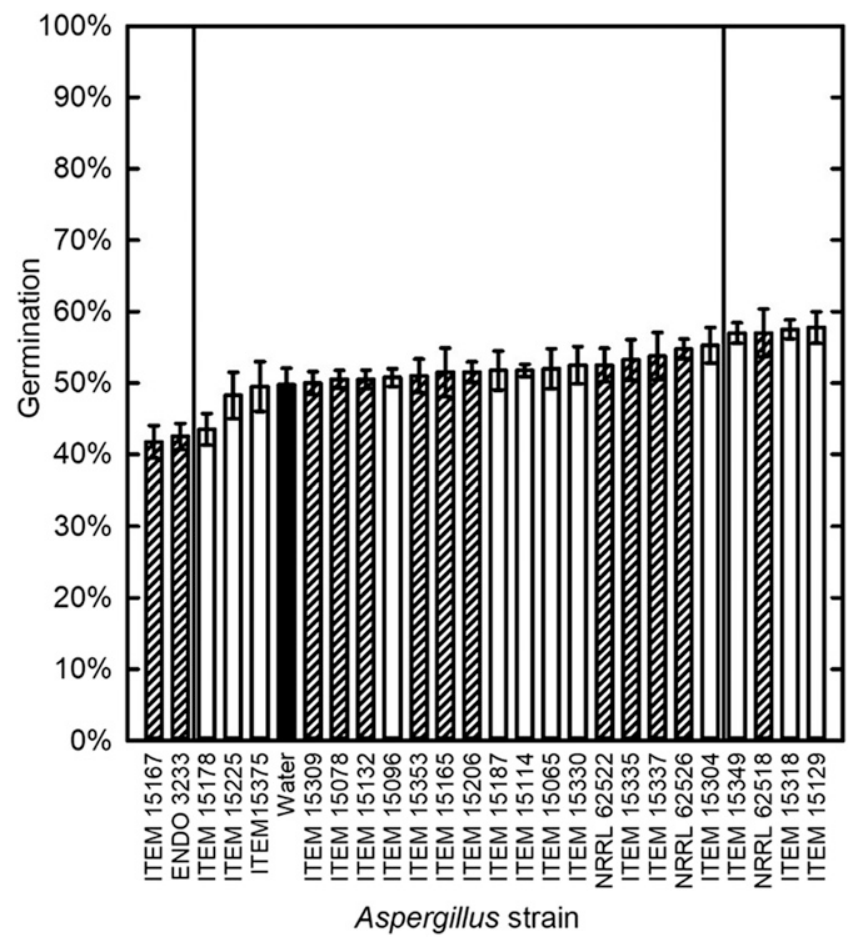

Fig. 4. Germination percentages in cold germination test for maize kernels (hybrid B) inoculated with strains of Aspergillus with and without production of fumonisin $\mathrm{B}_{2}$. Hatch marks indicate strains that have previously been documented to produce fumonisin $B_{2}$. Strains to the right of the vertical line were not significantly different from the water control $(\mathrm{LSD}=6.6 \%, \alpha=0.05)$. Error bars represent the standard error of the mean. 
For both maize hybrids, there were no significant differences in overall means for warm germination or cold test results between the fumonisin-producing Aspergillus strains and the nonproducing strains (hybrid A: $P=0.3893$ and $P=0.8225$; hybrid $\mathrm{B}: P=$ 0.1723 and $P=0.2058$ ). There were significant differences among species for effects on warm germination for hybrid A, and for cold germination for hybrid B. However, the only consistent pattern was significantly lower mean germination following inoculation with $A$. niger strains compared with A. welwitschiae and A. phoenicis.

Seedling disease assays. In the rolled-towel assays with hybrid A, there were significant differences among the U.S. strains for effects on root weight, shoot weight, and shoot length, but not for root length (Table 1). Root weight was numerically lower than the control for nine of the 10 strains, but only three strains, including two of the six fumonisin-producing strains, reduced root weight significantly (by up to $51 \%$ ) compared with the control. Eight strains significantly reduced shoot weight, including five fumonisin-producing strains. Nine of the 10 strains reduced shoot length, including five fumonisin-producing strains. There was little difference overall in the means for fumonisin-producing compared with nonproducing strains, and the effects of strains were greater for shoot variables than for roots. In fact, most of the strains had root length means that were numerically greater than (but not significantly different from) the control.

In the hybrid A assay with the Italian strains, there were significant differences among the strains in all four variables for maize hybrid A (Table 2). In root weight, all 11 of the strains differed significantly from the control, with one of the six fumonisin-producing strains (ITEM 15165) increasing the root weight by $19 \%$, and all other strains resulting in a decrease of between 17 and $45 \%$. Ten of the 11 strains reduced shoot weight, including five fumonisin-producing strains. Four strains increased root length, including two fumonisinproducing strains. Ten strains reduced shoot length by between 2 and $56 \%$, including five fumonisin-producing strains. Again, there was little difference overall in the means for fumonisin-producing compared with nonproducing strains; and shoot effects were greater than effects on roots. As with the U.S. strains, most had root length means that were numerically greater than the control, and this difference (up to a $27.7 \%$ increase) was significant for four strains (two fumonisin-producing and two nonproducing).

For hybrid B, there also were significant effects on all four variables (Table 3). Twenty-four of the 25 strains reduced root weight by between 9 and 22\%, including all 13 fumonisin-producing strains; 22 strains reduced shoot weight by between 13 and $22 \%$, including 12 fumonisin-producing strains; 13 strains reduced root length by between 2 and 19\%, including eight fumonisin-producing strains, and 21 of the 25 strains reduced shoot length by between 3 and $21 \%$, including 11 fumonisin-producing strains. Differences in overall means of the various measures of seedling growth between fumonisinproducing and nonproducing strains were not consistent. For hybrid A inoculated with U.S. strains (Table 1), there was no detectible difference between fumonisin-producing and nonproducing strains with regard to seedling root weight, shoot weight, root length, or shoot length ( $P$-values from 0.7303 to 0.8359$)$. However, among the Italian strains (Table 2), inoculation with fumonisin-producing strains resulted in significantly greater seedling shoot weight and length compared with the nonproducing strains $(P<0.05)$, with no difference in root weight or length $(P=0.3665$ and $P=0.6318$, respectively). With hybrid B (Table 3), inoculation with fumonisin-producing strains resulted in seedlings with decreased root and shoot weight compared with nonproducing strains ( $P=0.0313$ and $P=0.0363$, respectively). The root and shoot lengths were not significantly different $(P=0.1565$ and $P=0.1498$, respectively).

In the rolled-towel assays, effects on seedling growth did not differ consistently among Aspergillus species. For hybrid A inoculated with the U.S. strains (Table 1), A. phoenicis caused significantly lower root weight, shoot weight, and shoot length relative to $A$. welwitschiae, and root weight relative to A. niger. However, among the Italian strains (Table 2), there were no significant differences among species. On hybrid B (Table 3), A. niger reduced root weight relative to $A$. welwitschiae, and reduced shoot weight and length relative to both $A$. tubingensis and $A$. welwitschiae. Inoculation with $A$. phoenicis reduced shoot weight relative to $A$. tubingensis.

In all seedling disease assays, Aspergillus strains were recovered from each of the inoculated treatments, but not the control. Other fungi recovered from control seedlings were not identified.

Field experiments. Ear rot symptoms and signs typical of Aspergillus section Nigri occurred in inoculated treatments in all three experiments. In the Iowa experiments, ear rot symptoms were more severe in 2012 (maximum treatment mean of 28.1\%) than in 2013 (maximum 12.9\%). Ear rot severity was greater in the experiments with wound inoculations (overall means of 22.2 and $10.7 \%$ in 2012 and 2013, respectively) than in those with silk-channel inoculations (means of 4.6 and $4.8 \%$ in 2012 and 2013). Aspergillus strains caused ear rot symptoms with severity similar to that caused by the F. verticillioides strain. In 2012, ear rot severity for the five Aspergillus strains ranged from 16.1 to $28.1 \%$ in the wound inoculation treatments, and from 1.2 to $6.6 \%$ in the silk-channel inoculation treatments, and none of these means was significantly different from the $F$. verticillioides strain $(24.8 \%$ for wound inoculation and $5.2 \%$ for silk-channel inoculation) (Fig. 5A); in 2013, severity was significantly greater $(8.8 \%)$ for strain ITEM 15375 (A. niger, fumonisinnonproducing) than for $F$. verticillioides $(2.1 \%)$ for the silk-channel inoculation, whereas the other strains were not different from $F$. verticillioides (Fig. 5B). Ear rot severity in inoculated treatments was not always significantly different from the mock-inoculated control. In 2012, severity in the mock-inoculated control was $0.5 \%$ for silkchannel inoculation and $15.7 \%$ for wound inoculation, and the $F$. verticillioides treatment did not differ from the control for either inoculation method. However, two Aspergillus strains had greater severity than the control for each inoculation method (Fig. 5A). In

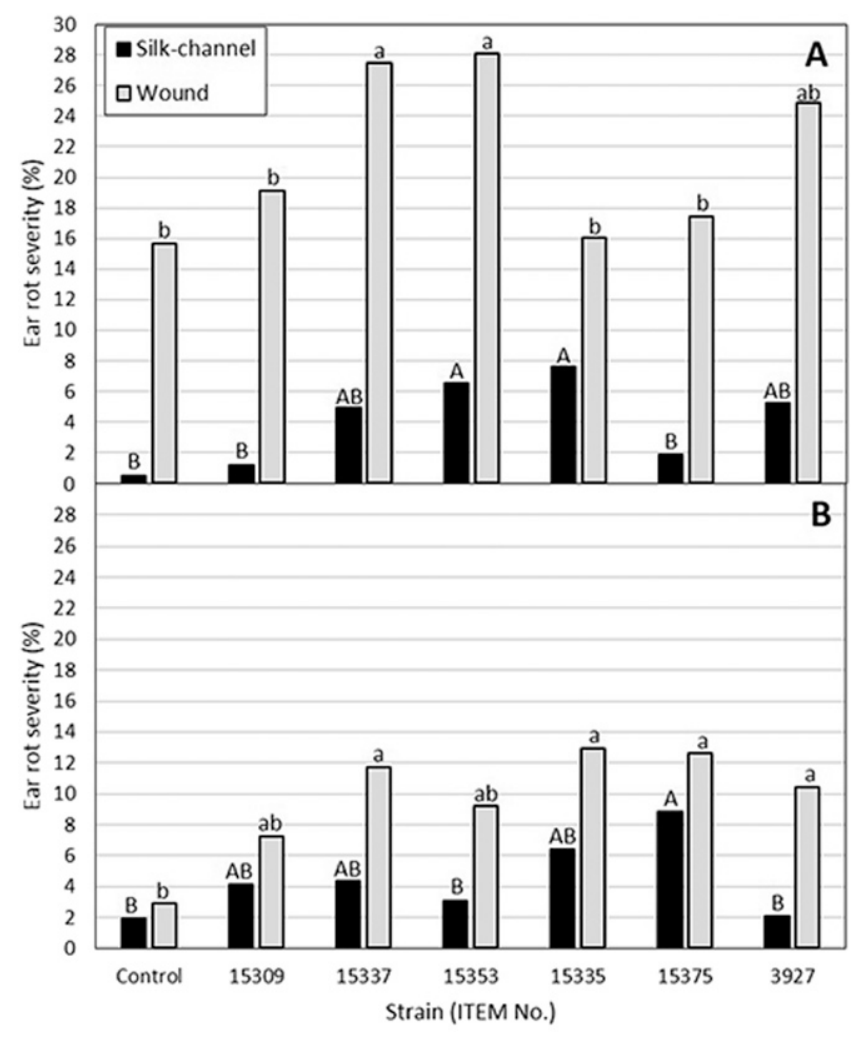

Fig. 5. Ear rot severity for maize ears inoculated with strains of Aspergillus section Nigri (ITEM15309 through ITEM15375) or Fusarium verticillioides (ITEM3927) in lowa field experiments in 2012 (A) and 2013 (B). Inoculation methods (silk-channel or wound) were applied in separate experiments. Data are transformed to percentages from original 1 to 7 disease severity scores. Capital letters indicate significant differences among strains inoculated by the silk-channel method; lowercase letters indicate significant differences among strains inoculated by the wound method, according to Fisher's protected least significant difference $(\alpha=0.05)$. 
2013, severity in the mock-inoculated control was $2.0 \%$ for silk-channel inoculation and $2.9 \%$ for wound inoculation. The $F$. verticillioides treatment had severity of $10.4 \%$ in the wound inoculation, which differed from the control, but in the silk-channel inoculation, the F. verticillioides strain also had severity of approximately $2.0 \%$. In 2013, one (ITEM 15375) and three Aspergillus strains (ITEM 15337,15335 , and 15375) differed from the control in the silkchannel inoculation and the wound inoculation, respectively. The fumonisin-nonproducing strain, ITEM 15375, differed from the control only in 2013, in both inoculation treatments (Fig. 5B); this strain caused less severe symptoms than two of the other Aspergillus strains in both inoculation treatments in 2012, but in 2013 it caused more severe symptoms than strain ITEM 15353 in the silk-channel treatment. Aspergillus strains were recovered from each of the symptomatic kernels sampled from inoculated treatments. From the control treatments, a few isolates of black Aspergillus were recovered, but most kernels from the control treatments yielded other fungi, which were not identified.
Fumonisin concentrations in grain were greatest in the $F$. verticillioides treatments in 2012 regardless of inoculation method, but the mock-inoculated, wounded control treatment was not significantly different from the $F$. verticillioides treatment (Table 4). In the silkchannel inoculation, concentrations of $\mathrm{FB}_{2}$ and total fumonisins did not differ between the control and the Aspergillus-inoculated treatments. The $\mathrm{FB}_{2} / \mathrm{FB}_{1}$ ratio (a measure of fumonisin production by Aspergillus spp.) was significantly greater for Aspergillus strains 15335 (ratio of 20.16) and 15337 (ratio of 23.84) compared with the other treatments, which ranged from 0.47 to 3.42 . In the wound inoculation, Aspergillus-inoculated treatments ranged from 3.09 to $10.89 \mu \mathrm{g} / \mathrm{g} \mathrm{FB}_{2}$ and from 15.87 to $28.57 \mu \mathrm{g} / \mathrm{g}$ total fumonisins, all of which were significantly lower than the $F$. verticillioides treatment and the control. The $\mathrm{FB}_{2} / \mathrm{FB}_{1}$ ratio was significantly greater for $A s$ pergillus strains 15337 (1.51) and 15353 (1.90) compared with the control (0.43), the $F$. verticillioides treatment (0.44), and Aspergillus strain 15375 (a fumonisin-nonproducing strain) (0.24) (Table 4). Fumonisins were lower in 2013 than in 2012, with a maximum

Table 4. Fumonisin contamination of grain from field experiments conducted in Iowa and Illinois following inoculation of maize ears with fumonisin-producing or nonproducing strains of Aspergillus ${ }^{\mathrm{v}}$

\begin{tabular}{|c|c|c|c|c|c|c|}
\hline Location-year & Inoculation & Strain $^{w}$ & Species & $F B_{2}(\mu g / g)$ & Total FB $(\mu \mathrm{g} / \mathrm{g})$ & Ratio $\left(\mathrm{FB}_{2} / \mathrm{FB}_{1}\right)$ \\
\hline \multirow[t]{14}{*}{ Iowa-2012 } & \multirow[t]{7}{*}{ Silk-channel } & Control & $\mathrm{NA}^{\mathrm{x}}$ & $0.13 \mathrm{~b}$ & $0.52 \mathrm{~b}$ & $0.61 \mathrm{~b}$ \\
\hline & & ITEM 15309 & A. welwitschiae & $0.51 \mathrm{~b}$ & $3.41 \mathrm{~b}$ & $0.44 \mathrm{~b}$ \\
\hline & & ITEM 15337 & A. niger & $0.87 \mathrm{~b}$ & $1.24 \mathrm{~b}$ & $23.84 \mathrm{a}$ \\
\hline & & ITEM 15353 & A. niger & $2.35 \mathrm{~b}$ & $3.82 \mathrm{~b}$ & $3.42 \mathrm{~b}$ \\
\hline & & ITEM 15335 & A. welwitschiae & $4.06 \mathrm{~b}$ & $4.99 \mathrm{~b}$ & $20.16 \mathrm{a}$ \\
\hline & & ITEM 15375 & A. niger & $1.02 \mathrm{~b}$ & $4.53 \mathrm{~b}$ & $0.32 \mathrm{~b}$ \\
\hline & & ITEM 3927 & F. verticillioides & $20.48 \mathrm{a}$ & $76.46 \mathrm{a}$ & $0.47 \mathrm{~b}$ \\
\hline & \multirow[t]{7}{*}{ Wound } & Control & NA & $37.59 \mathrm{a}$ & $141.35 \mathrm{a}$ & $0.43 \mathrm{~b}$ \\
\hline & & ITEM 15309 & A. welwitschiae & $10.89 \mathrm{~b}$ & $28.57 \mathrm{~b}$ & $0.90 \mathrm{ab}$ \\
\hline & & ITEM 15337 & A. niger & $9.53 \mathrm{~b}$ & $18.24 \mathrm{~b}$ & $1.51 \mathrm{a}$ \\
\hline & & ITEM 15353 & A. niger & $10.18 \mathrm{~b}$ & $26.28 \mathrm{~b}$ & $1.90 \mathrm{a}$ \\
\hline & & ITEM 15335 & A. welwitschiae & $8.15 \mathrm{~b}$ & $21.45 \mathrm{~b}$ & $0.96 a b$ \\
\hline & & ITEM 15375 & A. niger & $3.09 \mathrm{~b}$ & $15.87 \mathrm{~b}$ & $0.24 \mathrm{~b}$ \\
\hline & & ITEM 3927 & F. verticillioides & $42.15 \mathrm{a}$ & $159.27 \mathrm{a}$ & $0.44 \mathrm{~b}$ \\
\hline \multirow[t]{14}{*}{ Iowa-2013 } & \multirow[t]{7}{*}{ Silk-channel } & Control & NA & $1.53 \mathrm{a}$ & $3.99 \mathrm{a}$ & $0.64 \mathrm{a}$ \\
\hline & & ITEM 15309 & A. welwitschiae & $2.72 \mathrm{a}$ & $7.55 \mathrm{a}$ & $0.62 \mathrm{a}$ \\
\hline & & ITEM 15337 & A. niger & $3.55 \mathrm{a}$ & $8.67 \mathrm{a}$ & $0.77 \mathrm{a}$ \\
\hline & & ITEM 15353 & A. niger & $1.40 \mathrm{a}$ & $1.43 \mathrm{a}$ & NA \\
\hline & & ITEM 15335 & A. welwitschiae & $1.27 \mathrm{a}$ & $1.27 \mathrm{a}$ & NA \\
\hline & & ITEM 15375 & A. niger & $0.28 \mathrm{a}$ & $0.70 \mathrm{a}$ & $1.30 \mathrm{a}$ \\
\hline & & ITEM 3927 & F. verticillioides & $2.52 \mathrm{a}$ & $4.88 \mathrm{a}$ & $1.04 \mathrm{a}$ \\
\hline & \multirow[t]{7}{*}{ Wound } & Control & NA & $4.43 \mathrm{~b}$ & $12.25 \mathrm{~b}$ & $0.65 \mathrm{c}$ \\
\hline & & ITEM 15309 & A. welwitschiae & $8.05 \mathrm{~b}$ & $15.03 \mathrm{~b}$ & $8.61 \mathrm{a}$ \\
\hline & & ITEM 15337 & A. niger & $4.42 \mathrm{~b}$ & $6.67 \mathrm{~b}$ & $2.55 \mathrm{~b}$ \\
\hline & & ITEM 15353 & A. niger & $6.23 \mathrm{~b}$ & $9.16 \mathrm{~b}$ & $4.74 \mathrm{~b}$ \\
\hline & & ITEM 15335 & A. welwitschiae & $9.18 \mathrm{~b}$ & $13.57 \mathrm{~b}$ & $6.81 \mathrm{a}$ \\
\hline & & ITEM 15375 & A. niger & $4.88 \mathrm{~b}$ & $14.12 \mathrm{~b}$ & $0.86 \mathrm{c}$ \\
\hline & & ITEM 3927 & F. verticillioides & $15.86 \mathrm{a}$ & $36.35 \mathrm{a}$ & $0.84 \mathrm{c}$ \\
\hline \multirow[t]{12}{*}{ Illinois-2012 } & \multirow[t]{6}{*}{ Silk-channely } & Control & NA & 56.47 & 270.24 & 0.30 \\
\hline & & ITEM 15309 & A. welwitschiae & 4.92 & 6.44 & 13.90 \\
\hline & & ITEM 15337 & A. niger & 41.06 & 54.29 & 13.83 \\
\hline & & ITEM 15353 & A. niger & 68.07 & 90.04 & 12.55 \\
\hline & & ITEM 15333 & A. niger & 33.90 & 50.00 & 10.6 \\
\hline & & ITEM 15375 & A. niger & $\mathrm{ND}^{\mathrm{z}}$ & ND & NA \\
\hline & \multirow[t]{6}{*}{ Wound } & Control & NA & $9.81 \mathrm{ab}$ & $47.17 \mathrm{a}$ & $0.32 \mathrm{ab}$ \\
\hline & & ITEM 15309 & A. welwitschiae & $5.61 \mathrm{~b}$ & $17.98 \mathrm{a}$ & $0.56 \mathrm{ab}$ \\
\hline & & ITEM 15337 & A. niger & $1.36 \mathrm{~b}$ & $10.13 \mathrm{a}$ & $0.21 \mathrm{~b}$ \\
\hline & & ITEM 15353 & A. niger & $8.96 a b$ & $43.55 \mathrm{a}$ & $0.32 \mathrm{ab}$ \\
\hline & & ITEM 15333 & A. niger & $17.97 \mathrm{a}$ & $91.96 \mathrm{a}$ & $0.90 \mathrm{a}$ \\
\hline & & ITEM 15375 & A. niger & $1.47 \mathrm{~b}$ & $10.79 \mathrm{a}$ & $0.21 \mathrm{~b}$ \\
\hline
\end{tabular}

$\mathrm{v}$ Values represent means of four replicate field plots, except for the Illinois silk-channel inoculation experiment. Within each location-year and inoculation type, letters indicate significant differences according to Fisher's protected least significant difference $(\alpha=0.05)$.

${ }^{w}$ Strain numbers in bold indicate fumonisin $B_{2}$-producing strains; fumonisin-producing $F$. verticillioides (ITEM 3927) was included as a positive control in the Iowa experiments.

x NA: Not applicable.

${ }^{y}$ In the Illinois silk-channel inoculation experiment, grain from all four replicate plots was combined for fumonisin analysis; therefore, there are no statistical replications for these treatments.

${ }^{\mathrm{z}} \mathrm{ND}$ : Not detected. 
treatment mean of $36.35 \mu \mathrm{g} / \mathrm{g}$ total fumonisins in the $F$. verticillioides wound-inoculation treatment. In the silk-channel inoculations, $\mathrm{FB}_{2}$ concentrations ranged from 0.28 to $3.55 \mu \mathrm{g} / \mathrm{g}$, total fumonisins ranged from 0.70 to $8.67 \mu \mathrm{g} / \mathrm{g}$, and no treatments differed significantly from the control, at $1.53 \mu \mathrm{g} / \mathrm{g} \mathrm{FB}_{2}$ and $3.99 \mu \mathrm{g} / \mathrm{g}$ total fumonisins. In the 2013 wound inoculations, only the $F$. verticillioides treatment differed from the control in $\mathrm{FB}_{2}(15.86 \mu \mathrm{g} / \mathrm{g})$ and total fumonisins; concentrations in the control treatment were $4.43 \mu \mathrm{g} / \mathrm{g} \mathrm{FB}_{2}$ and $12.25 \mu \mathrm{g} / \mathrm{g}$ total fumonisins. The $\mathrm{FB}_{2} / \mathrm{FB}_{1}$ ratio was significantly greater for all four fumonisin-producing Aspergillus strains (ranging from 2.55 to 8.61 ) compared with the control, the $F$. verticillioides treatment, and the fumonisin-nonproducing $A s$ pergillus strain 15375 (ranging from 0.65 to 0.86 ) (Table 4).

In the Illinois experiment, the silk-channel inoculation resulted in more severe ear rot symptoms (overall mean 56.4\%) compared with the pipe-cleaner wounding inoculation method (30.8\%) (Fig. 6). In the silk-channel inoculation treatment, four of the five Aspergillus strains caused ear rot symptoms significantly more severe (up to 83.6\%) than in the control, which had mean ear rot severity of $1.5 \%$. In the wound inoculation treatment, only ITEM 15337 (A. niger), with severity of $43.2 \%$, was significantly different from the control $(24.3 \%)$. The fumonisin-nonproducing strain, ITEM 15375, showed severity of 67.4 and $31.0 \%$, respectively, in the silk-channel and pipe-cleaner inoculation treatments. These values differed from the control in the silk-channel inoculation treatment only, and were not significantly different from the other Aspergillus strains when inoculated using either method (Fig. 6).

In contrast to the Iowa results, fumonisin concentrations in grain were greatest in the silk-channel inoculation treatments, including the mock-inoculated control, which had a total fumonisin concentration exceeding $270 \mu \mathrm{g} / \mathrm{g}$ (Table 4). Although $\mathrm{FB}_{2} / \mathrm{FB}_{1}$ ratios were much higher in treatments inoculated with fumonisin-producing $A s$ pergillus strains (10.6 to 13.9) compared with the mock-inoculated control (0.30), statistical comparisons were not possible in the silkchannel inoculation treatments due to pooling of grain from the replicate plots. In the wound inoculations, the control had concentrations of $9.81 \mu \mathrm{g} / \mathrm{g} \mathrm{FB}_{2}$ and $47.17 \mu \mathrm{g} / \mathrm{g}$ total fumonisins; there were some significant differences among strains in fumonisin concentrations or $\mathrm{FB}_{2} / \mathrm{FB}_{1}$ ratios, but none differed significantly from the control (Table 4).

\section{Discussion}

The results of this study indicate that strains in Aspergillus section Nigri can be pathogenic to maize ears, germinating seeds, and seedlings. Although storage molds in general are associated with reduced emergence (Sauer et al. 1992) and seedling disease (Smith and White 1988), there are no previously published studies reporting the pathogenicity of Aspergillus section Nigri on maize seedlings. Windham and Williams (2012) reported on ear rot development following the inoculation of maize ears with $A$. niger, but they did not report effects on germination or seedling disease.

There was a wide range in both the warm and cold germination values among the Aspergillus strains examined. Results were variable between the warm and cold tests, and between the two hybrids, suggesting that the pathogenicity of these Aspergillus strains depends strongly on experimental conditions and host genotype. Other microorganisms already present in the kernels also may have influenced the results of the germination and rolled-towel assays. Although the kernels were surface-disinfested, it is likely that some kernels harbored other fungi or bacteria that were not eliminated by the surface treatment. Hot water treatment to further reduce endemic contamination may be advisable in order to reduce experimental variability. Hybrid B performed poorly, even in the absence of inoculation, suggesting poor quality seed. Results confirm that some strains of Aspergillus section Nigri can reduce germination and emergence in maize, but other strains have little capacity to do so.

Differences in aggressiveness among the Aspergillus species were not consistent. Among the species compared, A. niger inoculation often resulted in reduced germination, but results varied among experiments. The single strain of $A$. carbonarius examined did not demonstrate a high level of pathogenicity, often not differing from the control. However, little can be concluded about this species based on only one strain. In the rolled-towel assays, nearly all the strains of Aspergillus reduced shoot length and weight. Among the species examined, strains of A. phoenicis tended to result in shoot and root lengths and weights lower than the other species, but these differences were not always significant. Root length was less strongly affected than the other variables, and in some cases, root length actually increased with inoculation, even though root weight was reduced. It is possible that growth of lateral roots was reduced by some Aspergillus strains, causing more nutrients to be directed to lengthening the radicle and primary roots. If there are significant species differences in aggressiveness, a greater number of strains of each species may be required in order to detect this.

No clear relationship between fumonisin production and aggressiveness as a seedling pathogen was observed. One of the most

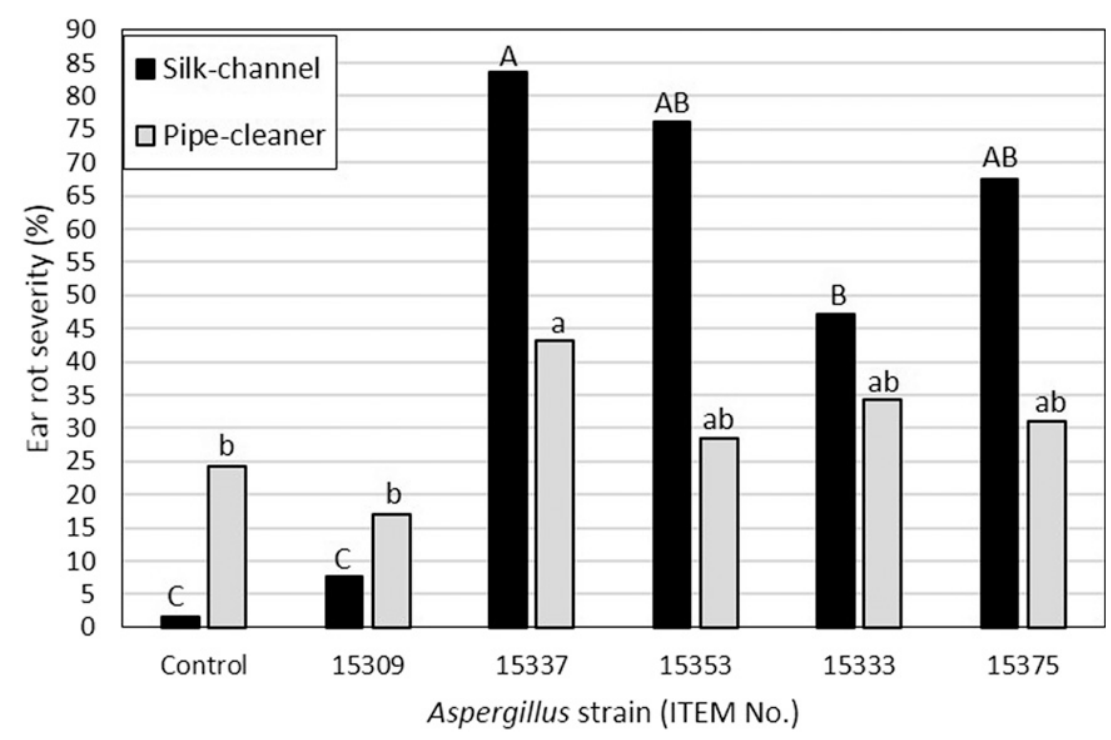

Fig. 6. Ear rot severity for maize ears inoculated with strains of Aspergillus section Nigri in an Illinois field experiment in 2012. Inoculation methods (silk-channel or wound) were applied in separate experiments. Data are transformed to percentages from original 1 to 7 disease severity scores. Capital letters indicate significant differences among strains inoculated by the silk-channel method; lowercase letters indicate significant differences among strains inoculated by the wound method, according to Fisher's protected least significant difference $(\alpha=0.05)$. 
aggressive strains overall was the fumonisin-nonproducing strain ITEM 15114, while fumonisin-producing strain 7 produced consistently low levels of symptoms. ITEM 15165, a fumonisinproducing strain, caused reductions in all seedling growth variables in hybrid B (Table 3), but hybrid A demonstrated increases in root weight and length when inoculated with ITEM 15165. This inconsistency cannot be easily explained, but does not alter the interpretation of the experiments. The lack of association between $\mathrm{FB}_{2}$ production and effects on germination for either hybrid indicates that fumonisin production is not necessary for, and probably has little or no role in the aggressiveness of black Aspergillus strains as seedborne pathogens. In the rolled-towel assays, there was some evidence for greater aggressiveness of fumonisin-producing strains toward hybrid B, but this was not consistent for hybrid A. For hybrid A, shoot length and weight were reduced to a greater extent by fumonisinnonproducing strains from Italy compared with the fumonisinproducing strains. This suggests that the differences observed were due to characteristics of the strains themselves, rather than their ability to produce fumonisins. It is also possible that hybrid $\mathrm{B}$ was more susceptible to $\mathrm{FB}_{2}$ than hybrid $\mathrm{A}$, though no effects of fumonisin production were evident for either hybrid in the germination tests.

The fact that inoculation with some strains resulted in greater emergence than the control in both cold tests may be due to competition with other pathogens in the media, as the sand used in the standard cold test is not sterile. It is also possible that other seedborne pathogens that are favored by cool conditions played a role. Weakly pathogenic strains of Aspergillus may have competed with pathogenic organisms already present in the sand, resulting in a net increase in germination.

Field experiments involved a smaller number of strains than the laboratory experiments, but results regarding pathogenicity, species comparisons, and the role of fumonisins were similar. There was no consistent difference in results between A. niger and A. welwitschiae, and the fumonisin-nonproducing strain produced ear rot severity similar to the other strains. Some strains of Aspergillus produced ear rot symptoms similar in severity to those caused by $F$. verticillioides, but other strains did not differ from the mock-inoculated control. Ear rot severity was in a similar range as reported by Windham and Williams (2012) for strains of A. niger. In Iowa in 2012, ear rot severity was relatively high in the mock-inoculated control for the wound treatment, and fumonisin concentrations for this treatment were greater than in the Aspergillusinoculated treatments. A similar observation occurred in the Illinois experiment, for both inoculation treatments. The inoculation procedures (which differed between the two locations) appeared to have promoted infection by other fungi present in the fields, particularly fumonisinproducing species of Fusarium. Sporulation consistent with $F$. verticillioides was observed on some ears from the mock-inoculated controls; however, no specific attempt was made to identify secondary fungi. Reduced concentrations of total fumonisins in the Aspergillus-inoculated treatments may have been the result of competition between the inoculated strains (which produce low concentrations of $\mathrm{FB}_{2}$ ) and endemic Fusarium strains (which include strains that produce high concentrations of fumonisin $\mathrm{B}_{1}, \mathrm{~B}_{2}$, and other forms). This impact was most evident in the case of fumonisin-nonproducing strain 15375 in Illinois, which had no detectable fumonisins in the silk-channel inoculation treatment, compared with $270.24 \mu \mathrm{g} / \mathrm{g}$ in the mock-inoculated control. Overall, ear rot severity and fumonisin concentrations were lower in 2013 compared with 2012 in Iowa, at least partially because late planting in 2013 resulted in altered ear morphology with most ear tips exposed beyond the husk leaves.

The Illinois results demonstrated severe ear rot symptoms and high fumonisin concentrations for the silk-channel inoculation. Environmental conditions differed between the two locations, and the use of an inbred line in Illinois, rather than a hybrid, may have contributed to the higher disease severity compared with that observed in Iowa. Results for the wound inoculation were similar between the locations in 2012, in spite of the use of different wounding procedures (method of Reid et al. [1996] in Iowa versus method of Dowell et al. [2002] in Illinois).
Inoculation with Aspergillus strains that produce $\mathrm{FB}_{2}$ did not result in increases in $\mathrm{FB}_{2}$ or total fumonisin concentrations in the grain, compared with the mock-inoculated control. However, the elevated $\mathrm{FB}_{2} / \mathrm{FB}_{1}$ ratio that resulted from these inoculations (Table 4 ) indicates that these strains were actively producing fumonisin $B_{2}$ in the infected kernels. This was not evident by comparing $\mathrm{FB}_{2}$ concentrations among the treatments, likely because Aspergillus strains produce small concentrations of $\mathrm{FB}_{2}$ relative to the concentrations produced by endemic Fusarium spp. The results of the field experiments indicate that fumonisin contamination of grain by strains of Aspergillus is probably minor in comparison with that caused by Fusarium spp.

Taken together, results of the field and laboratory experiments suggest that fumonisin-producing and nonproducing strains of Aspergillus have similar levels of aggressiveness on maize. Pathogenicity tests comparing wild-type fumonisin-producing Aspergillus strains against isogenic strains mutated by disruption of the fum 8 gene would provide a more definitive assessment of the role of fumonisin production in the pathogenicity of fungi in Aspergillus section Nigri. Several studies with fumonisin-producing species of Fusarium have concluded that there is little or no role of fumonisins in disease development (Covarelli et al. 2012); however, fumonisins are phytotoxic (Arias et al. 2012) and some researchers have concluded that fumonisins play a role in pathogenicity or virulence (Arias et al. 2012; Glenn et al. 2008; Sánchez-Rangel et al. 2012). Black Aspergillus species do not produce $\mathrm{FB}_{1}$, and overall the fumonisin production in these aspergilli is much lower than in fumonisin-producing species of Fusarium (Susca et al. 2014). If fumonisins can influence seedling disease, the effects may not be evident with the low concentrations of fumonisins produced by species of Aspergillus.

\section{Acknowledgments}

This research was conducted as part of the first author's Faculty Professional Development Assignment, supported by the Iowa State University College of Agriculture and Life Sciences and the Iowa Agricultural Experiment Station. The authors are grateful to the Italian Research Council (CNR) for use of facilities and other in-kind support at Istituto di Scienze delle Produzioni Alimentari (ISPA). The authors thank Derrick Mayfield (Iowa State University) and Angela Pastoressa (ISPA) for technical assistance.

\section{Literature Cited}

Arias, S. L., Theumer, M. G., Mary, V. S., and Rubinstein, H. R. 2012. Fumonisins: probable role as effectors in the complex interaction of susceptible and resistant maize hybrids and Fusarium verticillioides. J. Agric. Food Chem. 60: 5667-5675.

Association of Official Seed Analysts. 2014. AOSA Rules for Testing Seeds. Association of Official Seed Analysts, Washington, DC.

Covarelli, L., Stifano, S., Beccari, G., Raggi, L., Lattanzio, V. M. T., and Albertini, E. 2012. Characterization of Fusarium verticillioides strains isolated from maize in Italy: Fumonisin production, pathogenicity and genetic variability. Food Microbiol. 31:17-24.

Desjardins, A. E., Munkvold, G. P., Plattner, R. D., and Proctor, R. H. 2002. FUM1-a gene required for fumonisin biosynthesis but not for maize ear rot and ear infection by Gibberella moniliformis in field tests. Mol. PlantMicrobe Interact. 15:1157-1164.

Desjardins, A. E., Plattner, R. D., Nelsen, T. C., and Leslie, J. F. 1995. Genetic analysis of fumonisin production and virulence of Gibberella fujikuroi mating population A (Fusarium moniliforme) on maize (Zea mays) seedlings. Appl. Environ. Microbiol. 61:79-86.

Dowell, F. E., Pearson, T. C., Maghirang, E. B., Xie, F., and Wicklow, D. T. 2002 Reflectance and transmittance spectroscopy applied to detecting fumonisin in single corn kernels infected with Fusarium verticillioides. Cereal Chem. 79: 222-226.

Ellis, M. L., Broders, K. D., Paul, P. A., and Dorrance, A. E. 2011. Infection of soybean seed by Fusarium graminearum and effect of seed treatments on disease under controlled conditions. Plant Dis. 95:401-407.

Frisvad, J. C., Smedsgaard, J., Samson, R. A., Larsen, T. O., and Thrane, U. 2007. Fumonisin B2 production by Aspergillus niger. J. Agric. Food Chem. 55: 9727-9732.

Glenn, A. E., Zitomer, N. C., Zimeri, A. M., Williams, L. D., Riley, R. T., and Proctor, R. H. 2008. Transformation-mediated complementation of a FUM gene cluster deletion in Fusarium verticillioides restores both fumonisin production and pathogenicity on maize seedlings. Mol. Plant-Microbe Interact. 21:87-97.

Hong, S. B., Lee, M., Kim, D. H., Varga, J., Frisvad, J. C., Perrone, G., Gomi, K., Yamada, O., Machida, M., Houbraken, J., and Samson, R. A. 2013. Aspergillus 
luchuensis, an industrially important black Aspergillus in East Asia. PLoS One 8:e63769.

Koehler, B. 1959. Corn ear rots in Illinois. Bulletin 639, University of Illinois Agricultural Experiment Station, Urbana-Champaign, IL.

Månsson, M., Klejnstrup, M. L., Phipps, R. K., Nielsen, K. F., Frisvad, J. C., Gotfredsen, C. H., and Larsen, T. O. 2010. Isolation and NMR characterization of fumonisin B2 and a new fumonisin B6 from Aspergillus niger. J. Agric. Food Chem. 58:949-953.

McGee, D. C. 1988. Maize Diseases. A Reference Source for Seed Technologists. APS Press, St. Paul, MN.

Nielsen, K. F., and Logrieco, A. F. 2012a. Letter to the editor on Fumonisin contamination and fumonisin producing black Aspergilli in dried vine fruits of different origin, IJFM. 143:143-149. Int. J. Food Microbiol. 152:45.

Nielsen, K. F., and Logrieco, A. F. 2012b. Rebuttal to the response letter to our letter to the editor on 'Fumonisin contamination and fumonisin producing black Aspergilli in dried vine fruits of different origin. Int. J. Food Microbiol. 152:49-50.

Noonim, P., Mahakarnchanakul, W., Nielsen, K. F., Frisvad, J. C., and Samson, R. A. 2009. Fumonisin B2 production by Aspergillus niger in Thai coffee beans. Food Addit. Contam. 26:94-100.

Palencia, E. R., Hinton, D. M., and Bacon, C. W. 2010. The black Aspergillus species of maize and peanuts and their potential for mycotoxin production. Toxins (Basel) 2:399-416.

Prakash, R., and Jha, S. N. 2014. Basics of the genus Aspergillus. Int. J. Res. Bot. 4:26-30.

Reid, L. M., Hamilton, R. I., and Mather, D. E. 1996. Screening maize for resistance to Gibberella ear rot. Technical Bulletin 1996-5E, Agriculture and Agri-Food Canada, Ottawa, ON, Canada.

Rodriguez-Brljevich, C. 2008. Interaction of fungicide seed treatments and the Fusarium-maize (Zea mays L.) pathosystem. MS Thesis, Iowa State University, Ames, IA.

Sánchez-Rangel, D., Sánchez-Nieto, S., and Plasencia, J. 2012. Fumonisin B1, a toxin produced by Fusarium verticillioides, modulates maize $\beta$-1, 3-glucanase activities involved in defense response. Planta 235:965-978.
Sauer, D. B., Meronuck, R. A., and Christensen, C. M. 1992. Microflora. Pages 313-340 in: Storage of Cereal Grains and Their Products. D. B. Sauer, ed. American Association of Cereal Chemists, St. Paul, MN.

Shimizu, K., Nakagawa, H., Hashimoto, R., Hagiwara, D., Onji, Y., Asano, K., Kawamoto, S., Takahashi, H., and Yokoyama, K. 2015. The $\alpha$-oxoamine synthase gene fum 8 is involved in fumonisin $\mathrm{B}_{2}$ biosynthesis in Aspergillus niger. Mycoscience 56:301-308.

Smith, D. R., and White, D. G. 1988. Diseases of corn. Pages 687-766 in: Corn and Corn Improvement. G. F. Sprague and J. W. Dudley, eds. American Society of Agronomy, Madison, WI.

Susca, A., Moretti, A., Stea, G., Villani, A., Haidukowski, M., Logrieco, A., and Munkvold, G. 2014. Comparison of species composition and fumonisin production in Aspergillus section Nigri populations in maize kernels from USA and Italy. Int. J. Food Microbiol. 188:75-82.

Susca, A., Proctor, R. H., Mule, G., Stea, G., Ritieni, A., Logrieco, A., and Moretti A. 2010. Correlation of mycotoxin fumonisin B2 production and presence of the fumonisin biosynthetic gene fum8 in Aspergillus niger from grape. J. Agric. Food Chem. 58:9266-9272.

Varga, J., Frisvad, J. C., Kocsube, S., Brankovics, B., Toth, B., Szigeti, G., and Samson, R. A. 2011. New and revisited species in Aspergillus section Nigri. Stud. Mycol. 69:1-17

Varga, J., Kocsubé, S., Suri, K., Szigeti, G., Szekeres, A., Varga, M., Tóth, B., and Bartók, T. 2010. Fumonisin contamination and fumonisin producing black Aspergilli in dried vine fruits of different origin. Int. J. Food Microbiol. 143: 143-149.

Varga, J., Kocsubé, S., Tóth, B., and Bartók, T. 2012. Response to letter to the editor on Fumonisin contamination and fumonisin producing black Aspergilli in dried vine fruits of different origin published in International Journal of Food Microbiology, 143:143-149. Int. J. Food Microbiol. 152:46-48.

Windham, G., and Williams, W. 2012. Comparison of different inoculating methods to evaluate the pathogenicity and virulence of Aspergillus niger on two maize hybrids. Phytoparasitica 40:305-310. 